\title{
Influence of Band Structure and Interatomic Exchange on Spin Fluctuations in Metals
}

\section{Schrieffer, John Roberts}

Published in:

Physical Review Letters

Link to article, DOI:

10.1103/PhysRevLett.19.644

Publication date:

1967

Document Version

Publisher's PDF, also known as Version of record

Link back to DTU Orbit

Citation (APA):

Schrieffer, J. R. (1967). Influence of Band Structure and Interatomic Exchange on Spin Fluctuations in Metals. Physical Review Letters, 19(11), 644-647. https://doi.org/10.1103/PhysRevLett.19.644

\section{General rights}

Copyright and moral rights for the publications made accessible in the public portal are retained by the authors and/or other copyright owners and it is a condition of accessing publications that users recognise and abide by the legal requirements associated with these rights.

- Users may download and print one copy of any publication from the public portal for the purpose of private study or research.

- You may not further distribute the material or use it for any profit-making activity or commercial gain

- You may freely distribute the URL identifying the publication in the public portal 
the crystal current. That such an effect exists is also consistent with the stated observations on the well-formed electric field domains.

That is, a limiting velocity of the charge carriers, and a decrease in that velocity with an increase in the electric field, would be a sufficient mechanism to stabilize the width of the domains.

With a polychromatic polarized probing light the bands appear as alternate colors. The photoinduced region of the static effect previously described also displays a different color from the unaffected area of the sample in a white probing light. The various colors illustrate the dispersion inherent in optically active materials. This very effectively demonstrates the differences in polarization of adjacent areas.

We wish to thank G. J. Kominiak for his very able technical assistance, and G. G. Douglas and R. N. Zitter for discussions on photoconductivity.

\footnotetext{
${ }^{1}$ A. A. Ballman, J. Crystal Growth 1, 37 (1967).

${ }^{2}$ P. V. Lenzo, E. G. Spencer, and A. A. Ballman, Appl. Opt. $\underline{5}, 1688$ (1966).

${ }^{3} \mathrm{~A}$. W. Warner and M. Onoe, to be published.

${ }^{4}$ G. G. Douglas and R. N. Zitter, to be published.

${ }^{5}$ E. G. Spencer, P. V. Lenzo, and A. A. Ballman, Appl. Phys. Letters $\underline{9}, 290$ (1966).

${ }^{6}$ K. W. Böer and W. E. Wilhelm, Phys. Status Solidi 4, 237 (1964).

${ }^{7}$ D。C. Northrup, P。 R. Thornton, and K. E. Trezise, Solid State Electron. 7, 17 (1964).

${ }^{8}$ Handbuch der Physik, edited by S. Flügge (SpringerVerlag, Berlin, Germany, 1961), Vol. 25, Pt. I. J. F. Nye, Physical Properties of Crystals (Oxford University Press, Oxford, England, 1960), p. 266.

${ }^{9}$ B. R. Ridley and J. B. Watkins, Proc. Phys. Soc. (London) 78, 293 (1961).
}

\title{
INFLUENCE OF BAND STRUCTURE AND INTERATOMIC EXCHANGE ON SPIN FLUCTUATIONS IN METALS
}

\author{
J. R. Schrieffer*† \\ Technical University, Lyngby, Denmark, and Institute of Experimental Physics, Geneva, Switzerland \\ (Received 26 June 1967)
}

Theoretical work of Berk and Schrieffer ${ }^{1}$ and of Doniach and Engelsberg ${ }^{2}$ has suggested the importance of electron-paramagnon (spinfluctuation) interactions in metals which are nearly ferromagnetic. Recent experiments on Ni-Rh alloys by Bucher et al. ${ }^{3}$ and on $\mathrm{Ni}-$ Pd alloys by Schindler ${ }^{4}$ give striking confirmation of these effects.

Initially, the theory was carried out for a spherical one-band model with a contact-exchange interaction. This model predicts exceptionally large many-body corrections, e.g., a mass enhancement of order 8 for Pd. From augmented-plane-wave ${ }^{5}$ and relativistic augmented-plane-wave (RAPW) ${ }^{6}$ calculations on $\mathrm{Pd}$, and the observed specific heat, one finds a factor closer to 2 . In addition, the alloy experiments exhibit a considerably smaller mass enhancement than this model predicts. In an attempt to account for this discrepancy, Doni$\mathrm{ach}^{7}$ added Hund's rule (intra-atomic) exchange to the model and found a reduction of the predicted enhancement, although it appears that the reduction is insufficient to bring theory and experiment into agreement. ${ }^{3}$

We report here on the importance of (a) band- structure effects and (b) interatomic exchange interactions in the problem, and show that they can lead to large additional changes in the manybody corrections.

Band-structure calculations ${ }^{5,6}$ show that the heavy-hole energy contours in Pd can be roughly represented by three cylindrical sub-bands, each of length equal to the (100) reciprocal lattice vector and having their axes along $x, y$, and $z$, respectively. The Bloch functions $\psi_{k \alpha}$ for these sub-bands can be represented in terms of Wannier functions $w_{\alpha}$ by

$$
\begin{gathered}
\psi_{k \alpha}=(N)^{-1 / 2} \sum_{j} \exp \left(i \overrightarrow{\mathrm{k}} \cdot \overrightarrow{\mathrm{R}}_{j}\right) w_{\alpha}\left(\overrightarrow{\mathrm{r}}-\overrightarrow{\mathrm{R}}_{j}\right), \\
\alpha=(x, y, z),
\end{gathered}
$$

where $N$ is the number of cells in the crystal. Recent theoretical work ${ }^{8}$ shows that for $d$ bands, the $w_{\alpha}$ behave like tight binding functions, and we expect matrix elements of short-ranged (screened) potentials taken between $w$ 's located on different sites will decrease rapidly with the site separation. Thus, we expand the Bloch matrix element $\left\langle\vec{p}+\vec{q}, \alpha ; \vec{k}, \beta^{\prime}|V| \vec{k}+\vec{q}, \beta ; \vec{p}, \alpha\right\rangle$ in Wannier functions and retain only the oneand two-center terms. The importance of in- 
ter-atomic exchange in $\mathrm{Pd}$ has been stressed recently by Clogston ${ }^{9}$ and by Peter. ${ }^{10}$ If $\alpha$ denotes $w_{\alpha}$ centered at the origin and $\alpha_{\rho}$ denotes $w_{\alpha}$ centered at $\vec{\rho}$, the relevant contributions to $N$ times the matrix element are the one-center (intra-atomic) terms

$$
\begin{aligned}
& \langle\alpha \alpha|V| \alpha \alpha\rangle=U \quad \text { (self-exchange), } \\
& \langle\beta \alpha|V| \alpha \beta\rangle=J_{\mathrm{H}^{\prime}} \quad \alpha \neq \beta
\end{aligned}
$$

(Hund's rule exchange),

and the two-center (interatomic terms

$$
\begin{gathered}
\sum_{\vec{\rho} \neq 0}\left\langle\alpha \beta_{\rho}|V| \alpha \beta_{\rho}\right\rangle e^{i(\overrightarrow{\mathrm{p}}-\overrightarrow{\mathrm{k}}) \cdot \vec{\rho}}=U_{\alpha \beta}{ }^{\prime}(\overrightarrow{\mathrm{p}}-\overrightarrow{\mathrm{k}}), \\
\sum_{\vec{\rho} \neq 0}\left\langle\beta_{\rho} \alpha|V| \alpha \beta_{\rho}\right\rangle e^{i \overrightarrow{\mathrm{q}} \cdot \vec{\rho}}=J_{\alpha \beta^{\prime}}(\overrightarrow{\mathrm{q}}) .
\end{gathered}
$$

We neglect one-body hopping terms, e.g., $\left\langle\alpha \alpha_{\rho}|V| \alpha \alpha\right\rangle$ since they are already included in the band-structure calculation.

Central to the spin-fluctuation theory ${ }^{1,2}$ is the spin-one particle-hole $t$ matrix $t_{p k} \alpha \beta(q)$ describing a particle-hole pair of total fourmomentum $q$ scattering between bands $\alpha$ and $\beta$. The self-energy (and hence the effective mass) and the spin susceptibility are simply related to this quantity. The integral equation for $t$ is

$$
\begin{aligned}
& t \underset{\mathrm{pk}}{\rightarrow \vec{k}} \alpha \beta(q) \\
& =V_{\overrightarrow{\mathrm{pk}}} \alpha \beta+\sum_{\alpha^{\prime}} \int V_{\overrightarrow{\mathrm{pp}}^{\prime}} \alpha \alpha^{\prime} K_{\overrightarrow{\mathrm{p}}^{\prime}} \alpha_{t \rightarrow \overrightarrow{\mathrm{p}}^{\prime} \mathrm{k}}^{\prime} \alpha^{\prime} \frac{d^{3} p^{\prime}}{(2 \pi)^{3}},
\end{aligned}
$$

where we have suppressed $q$ and set

$$
\begin{gathered}
V_{p k}{ }^{\alpha \beta}(q) \\
=\left(U-J_{\mathrm{H}}\right) \delta_{\alpha \beta}+J_{\mathrm{H}}+U_{\alpha \beta^{\prime}}(\overrightarrow{\mathrm{p}}-\overrightarrow{\mathrm{k}})+J_{\alpha \beta^{\prime}}(\overrightarrow{\mathrm{q}}), \\
K_{\overrightarrow{\mathrm{p}}}^{\alpha}(q)=i \int G^{\alpha}(p+q) G^{\alpha}(p) \frac{d p_{0}}{2 \pi},
\end{gathered}
$$

with $G^{\alpha}$ being the one-particle Green's function for sub-band $\alpha . K_{p}{ }^{\alpha}(q)$ is related to the zero-order susceptibility (in units of $2 \mu_{\mathrm{B}}{ }^{2}$, $\mu_{\mathrm{B}} \equiv$ Bohr magneton) for sub-band $\alpha$ by $^{11}$

$$
\chi_{0}^{\alpha}(q)=\int K_{\overrightarrow{\mathrm{p}}}^{\alpha}(q) \frac{d^{3} p}{(2 \pi)^{3}}
$$

Umklapp processes are included by working with a repeated-zone scheme in (4) and restricting $\vec{p}$ to the first Brillouin zone. The paramagnon contribution to the mass enhancement is given in terms of the static $\left(q_{0}=0\right) t$ matrix by $^{12}$

$$
\begin{aligned}
& \frac{\delta m_{\overrightarrow{\mathrm{k}}} \alpha}{m_{\overrightarrow{\mathrm{k}}} \alpha} \\
& =\int\left[t_{\overrightarrow{\mathrm{k}} \overrightarrow{\mathrm{k}}} \alpha \alpha\left(\overrightarrow{\mathrm{k}}-\overrightarrow{\mathrm{k}}^{\prime}\right)-V_{\overrightarrow{\mathrm{k}} \overrightarrow{\mathrm{k}}} \alpha \alpha\left(\overrightarrow{\mathrm{k}}-\overrightarrow{\mathrm{k}}^{\prime}\right)\right] \delta\left(\epsilon_{\overrightarrow{\mathrm{k}}^{\prime}} \alpha\right) \frac{d^{3} k^{\prime}}{(2 \pi)^{3}},
\end{aligned}
$$

while the dynamic susceptibility is

$$
\chi(q)=\sum_{\alpha} \chi_{0}^{\alpha}(q)+\sum_{\alpha \beta \overrightarrow{\mathrm{pk}}} K_{\overrightarrow{\mathrm{p}}}^{\alpha}(q) t_{\overrightarrow{\mathrm{pk}}}^{\alpha \beta} K_{\overrightarrow{\mathrm{k}}}^{\beta}(q)
$$

By neglecting the interatomic interactions $U^{\prime}$ and $J^{\prime}$, and considering for the moment three spherical sub-bands, one obtains the Hund's rule model of Doniach, ${ }^{7}$ for which the solution of $(3 a)$ is

$$
t_{D}^{\alpha \beta}(q)=\left(U-J_{\mathrm{H}}\right) F(q) \delta_{\alpha \beta}+\frac{J_{\mathrm{H}} F(q)^{2}}{1-3 J_{\mathrm{H}} \chi_{0}(q) F(q)} .
$$

Here, the enhancement factor

$$
F^{\alpha}(q)=\frac{1}{1-\left(U-J_{\mathrm{H}}\right) \chi_{0}^{\alpha}(q)}
$$

is independent of $\alpha$ because of the spherical sub-band assumption.

More generally, if one adds band-structure effects and the interatomic exchange $J^{\prime}(q)$ (but not $U^{\prime}$ ), one obtains

$$
\begin{array}{r}
t_{(q)=\left(U-J_{\mathrm{H}}\right) F^{\alpha}(q) \delta_{\alpha \beta}} \\
+\frac{J(q) F^{\alpha}(q) F^{\beta}(q)}{1-J(q) \sum_{\alpha^{\prime}} \chi_{0}^{\alpha^{\prime}}(q) F^{\alpha^{\prime}}(q)}
\end{array}
$$

where

$$
J(q)=J_{\mathrm{H}}+J^{\prime}(q) .
$$

We consider first the spherical sub-band case, and decompose $t^{\alpha \beta}$ into its three spinfluctuation eigenmodes, ${ }^{7}$

$$
\begin{aligned}
& t^{\alpha \beta}(q)=\frac{1}{3} \frac{U+2 J_{\mathrm{H}}+3 J^{\prime}(q)}{1-\left[U+2 J_{\mathrm{H}}+3 J^{\prime}(q)\right] \chi_{0}(q)}
\end{aligned}
$$

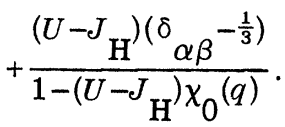


The first term, corresponding to an in-phase spin fluctuation of the sub-bands, is influenced by the interatomic exchange $J^{\prime}$, while the last term, arising from antiphase modes, is inde-

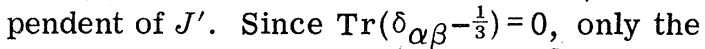
in-phase mode contributes to $\chi(q)$ [see Eq. 5(b)]:

$$
\chi(q)=\frac{3 \chi_{0}(q)}{1-\left[U+2 J_{\mathrm{H}}+3 J^{\prime}(q)\right] \chi_{0}(q)} \equiv 3 \chi_{0}(q) S(q)
$$

The Stoner factor $S(0)$ is roughly of order 20 for Pd. For $J_{\mathrm{H}}=J^{\prime}=0$, all three modes contribute equally to $\delta m / m$. For $J^{\prime}(q)=0$, the inphase mode continues to contribute one-third of the $J_{\mathrm{H}}=0$ mass shift. However, for $J^{\prime}(q)$ $\neq 0$ the decrease of $J^{\prime}$ as $q$ increases from zero, Eq. (2d), leads to a more rapid drop of the in-phase part of $t(q)$ for small $q$ and hence to a smaller mass correction. For $J_{H}$ and/or $J^{\prime}$ nonzero, the antiphase mode contribution to the mass correction is reduced because of the reduced exchange coupling for these modes, i.e., $U-J_{\mathrm{H}}$ rather than $U+2 J_{\mathrm{H}}+3 J^{\prime}$.

These effects are illustrated in Fig. 1, where $\delta \mathrm{m} / \mathrm{m}$ is plotted for spherical sub-bands with (a) $J^{\prime}=0$ (Hund's rule model) and $(b) J_{H}=0$ (interatomic exchange model) as a function of the exchange strength. The Stoner factor, $S(0)$

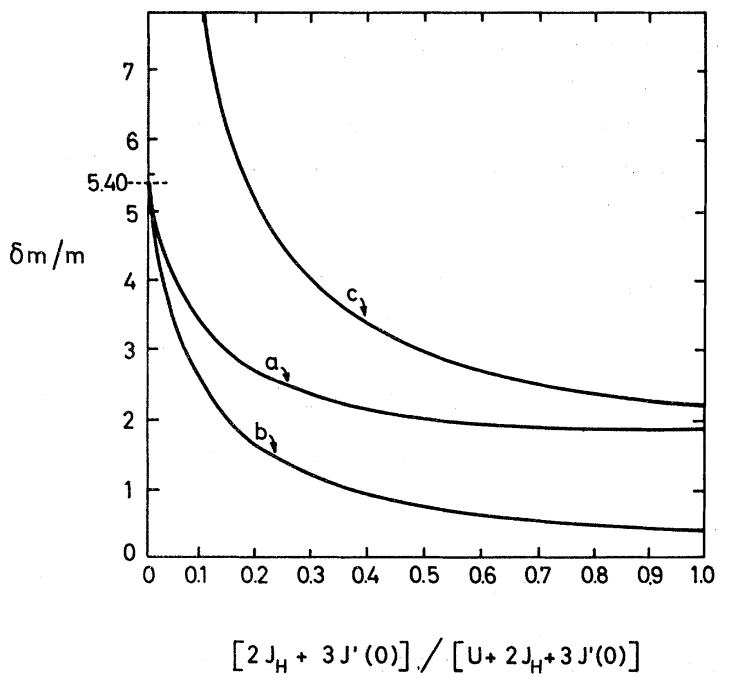

FIG. 2. The paramagnon contribution to the mass enhancement factor for three limiting models: $a$, Hund's rule $\left(J_{\mathrm{H}}\right)$ and self-exchange $(U)$ with spherical subbands; $b$, interatomic $\left(J^{\prime}\right)$ and self-exchange with spherical sub-bands; and $c$, Hund's rule and self-exchange for cylindrical sub-bands. The results are plotted as a function of the fraction of the non- $U$ exchange entering the Stoner factor, i.e., $\left(2 J_{\mathrm{H}}+3 J^{\prime}\right) /$ $\left(U+2 J_{\mathrm{H}}+3 J^{\prime}\right)$, with the Stoner factor chosen to be 20 . of (9), was set equal to 20 . We assumed $J^{\prime}(q)$ $=J^{\prime}\left(1-q^{2} a^{2} / 6\right)$, where $a$ is the nearest-neighbor distance, chosen such that $k_{\mathrm{F}} a=1.7$. We see that interatomic exchange can be much more effective than Hund's rule exchange in reducing $\delta m / m$. For example, $m^{*} / m$ is 2.2 if $30 \%$ of the exchange entering the Stoner factor is interatomic in origin, while it is 3.4 for this strength of Hund's rule exchange. ${ }^{13}$

Turning to band-structure effects, we plot in Curve $c$ the results for the cylinder model with $J^{\prime}=0$. For a cylindrical sub-band, $\chi_{0}{ }^{\alpha}(q)$ is independent of $\vec{q}$ if $\vec{q}_{\perp}$ (the component of $q$ perpendicular to the cylinder axis) is less than the cylinder diameter. Therefore, $t$ remains large for a large range of $\vec{q}$ about $q=0$ and a large mass enhancement results. Judging from the RAPW plots of the Fermi surface of $\mathrm{Pd}{ }^{6}$ the cylinder model overemphasized the weak $\vec{q}$ variation of $\chi_{0}(q)$ for small $q$ and some compromise between the results of the spherical and cylindrical models seems appropriate.

We note that the two-center counterpart $U^{\prime}(\overrightarrow{\mathrm{p}}$ $-\overrightarrow{\mathrm{k}}$ ) of the self-exchange $U$ may in fact be the dominant interatomic exchange coupling, judging from the matrix elements $(2 \mathrm{c})$ and $(2 \mathrm{~d})$, i.e., "direct" versus "exchange." Preliminary analysis indicates the effects of $U^{\prime}$ are similar to those of $J^{\prime}$. As was recently stressed, ${ }^{9,10}$ the neutron-scattering experiments of Low ${ }^{14}$ on dilute $\mathrm{Fe}$ and $\mathrm{Co}$ in $\mathrm{Pd}$ seem to require a major fraction of the exchange in $\mathrm{Pd}$ to be interatomic in character. ${ }^{15}$ If this is the case, it appears from the above results that the discrepancy between the predicted paramagnon contribution to the mass enhancement and the modest total enhancement (including the phonon contribution) required by band-structure analysis ${ }^{6}$ may be resolved in this way. It is possible that interatomic exchange also plays an important role in reducing $\delta \mathrm{m} / \mathrm{m}$ in the alloy experiments..$^{3,4}$ Neutron-scattering studies of $\chi(q)$ in these systems, similar to those mentioned above, would be helpful in settling this question.

In addition to reducing the mass enhancement, interatomic exchange reduces the spin-fluctuation contribution to the pairing interaction in superconductors. In the absence of pseudopotential effects (which were shown to be small for the one-band contact exchange $\operatorname{model}^{1}$ ), one has $N(0) V_{\text {spin }}=\delta m / m$. Whether this reduced spin-fluctuation repulsion dominates the Coulomb repulsion in suppressing supercon- 
ductivity at the right-hand end of the transition metal series is not clear at present.

The author would like to thank Dr. A. R. Mackintosh, Dr. O. K. Andersen, Dr. M. Peter, Dr. B. Giovannini, Dr. E. Bucher, Dr. A. I. Schindler, Dr. A. M. Clogston, Dr. W. F. Brinkman, Dr. W. Brenig, Dr. S. Doniach, and Dr. N. F. Berk for interesting discussions and correspondence on these questions. The author is also indebted to Dr. E. Bucher et al. for sending a copy of their manuscript prior to publication. The hospitality of the Laboratory for Electrophysics of the Technical University of Denmark and the Laboratory of Experimental Physics of the University of Geneva is gratefully acknowledged.

\footnotetext{
*Permanent address: Department of Physics, University of Pennsylvania, Philadelphia, Pennsylvania.

†John Simon Guggenheim Fellow, 1966-1967.

${ }^{1}$ N. F. Berk and J. R. Schrieffer, Phys. Rev. Letters 17, 433 (1966); Proceedings of the Tenth International Conference on Low Temperature Physics, Moscow, 31 August-6 September 1966 (to be published). N. F. Berk, thesis, University of Pennsylvania, Philadelphia, Pennsylvania, 1966 (unpublished).

${ }^{2}$ S. Doniach and S. Engelsberg, Phys. Rev. Letters 17, 750 (1966); S. Doniach, S. Engelsberg, and M. I. Rice, in Proceedings of the Tenth International Conference on Low Temperature Physics, Moscow, $31 \mathrm{Au}-$
}

gust-6 September 1966 (to be published).

${ }^{3}$ E. Bucher, W. F. Brinkman, J. P. Maita, and H. J. Williams, Phys. Rev. Letters 18, 1125 (1967).

${ }^{4}$ A. I. Schindler, private communication.

${ }^{5}$ A. J. Freeman, J. O. Dimmock, and A. M. Furdyna, J. Appl. Phys. 37, 1256 (1965).

${ }^{6}$ O. K. Andersen and A. R. Mackintosh, to be published.

${ }^{7}$ S. Doniach, Phys. Rev. Letters 18, 554 (1967).

${ }^{8} \mathrm{~V}$. Heine, Phys. Rev. 153, 673 (1967), and further references therein.

${ }^{9}$ A. M. Clogston, private communication.

${ }^{10} \mathrm{M}$. Peter, private communication.

${ }^{11} \mathrm{~J}$. R. Schrieffer and N. F. Berk, Phys. Letters 24A, 604 (1967). It is shown here that one can use bare $\overline{G ' s}$ in this expression near the ferromagnetic instability.

${ }^{12}$ It has been argued by W. Brenig and by W. F. Brinkman that the integral (5a) should be multiplied by a factor of $\frac{3}{2}$ to account for the $m_{z}=0$ component of the (spin-one) spin fluctuations (private communication).

${ }^{13}$ It has been independently suggested by Bucher, Brinkman, Maita, and Williams that $q$-dependent exchange may play a role in reducing the predicted mass enhancement (see Ref. 3).

${ }^{14} \mathrm{G}$. G. Low, in Proceedings of the International Conference on Magnetism, Nottingham, England, 1964 (The Institute of Physics and the Physical Society, London, England, 1965), p. 133.

${ }^{15}$ Since a set of well-localized Wannier functions (1) will in general depend on both $k$ and $\alpha$, even the onecenter terms will be momentum dependent. These effects can further reduce the mass enhancement and increase the range of $\chi(\boldsymbol{r})$. See J. R. Schrieffer, to be published.

\title{
NUCLEAR RELAXATION IN SUPERCONDUCTING NIOBIUM NEAR THE UPPER CRITICAL FIELD
}

\author{
M. Cyrot, C. Froidevaux, and D. Rossier \\ Service de Physique des Solides, Laboratoire associé au Centre National de la Recherche \\ Scientifique, Faculté des Sciences, Orsay, France
}

(Received 24 July 1967)

Up to now most nuclear-relaxation-time measurements in the mixed state of Type-II superconductors have been made far below the upper critical field $H_{c 2}$. Their interpretation generally involves the well-known BCS behavior. ${ }^{1}$ We report here $T_{1}$ measurements in pure niobium near $H_{C 2}$. The new point is essentially that in this region the distance between vortices becomes comparable with the coherence length. This is a gapless situation, ${ }^{2}$ which gives rise to new features in the nuclear relaxation.

$T_{1}$ was measured in two different samples of niobium powder with average particle of 15 $\mu$. We label them $\mathrm{Nb} \mathrm{I}$ and $\mathrm{Nb}$ II. The upper critical fields at zero temperature are, respectively, $H_{c 2 \mathrm{I}}=8.2 \mathrm{kG}$ and $H_{c 2 \mathrm{II}}=12.1 \mathrm{kG}$, as determined by extrapolating the variation of $H_{c 2}$ vs $T$. By using the theoretical electron mean free path dependence ${ }^{3}$ of the upper critical field of a Type-II superconductor, the ratio of the coherence length $\xi_{0}$ to the mean free path $l$ is found to be $\xi_{0} / l=0.7$ for $\mathrm{Nb} \mathrm{I}$ and $\xi_{0} / l$ $=1.5$ for Nb II. Thus it turns out that sample I is rather clean, while sample II is rather dirty, though the $\xi_{0} / l$ parameters differ by only a small factor. Most of the $T_{1}$ measurements were done at temperatures between 1.4 and $4.2^{\circ} \mathrm{K}$, in magnetic fields from 3 to $9 \mathrm{kG}$ (namely $0.15 \leqslant T / T_{c} \leqslant 0.45$ and $0.4 \leqslant H / H_{c 2} \leqslant 1$ ). $T_{1}$ was measured at the center of the nuclear magnetic resonance line in the following way: We saturated the thermal-equilibrium nuclear mag- 